\title{
The association of lung function and St. George's respiratory questionnaire with exacerbations in COPD: a systematic literature review and regression analysis
}

Amber L. Martin ${ }^{1 *}$, Jessica Marvel ${ }^{2}$, Kyle Fahrbach', Sarah M. Cadarette ${ }^{1}$, Teresa K. Wilcox ${ }^{1}$ and James F. Donohue ${ }^{3}$

\begin{abstract}
Background: This study investigated the relationship between changes in lung function (as measured by forced expiratory volume in one second $\left[\mathrm{FEV}_{1}\right]$ ) and the St. George's Respiratory Questionnaire (SGRQ) and economically significant outcomes of exacerbations and health resource utilization, with an aim to provide insight into whether the effects of COPD treatment on lung function and health status relate to a reduced risk for exacerbations.

Methods: A systematic literature review was conducted in MEDLINE, Embase, and the Cochrane Central Register of Controlled Trials to identify randomized controlled trials of adult COPD patients published in English since 2002 in order to relate mean change in $\mathrm{FEV}_{1}$ and SGRQ total score to exacerbations and hospitalizations. These predictor/ outcome pairs were analyzed using sample-size weighted regression analyses, which estimated a regression slope relating the two treatment effects, as well as a confidence interval and a test of statistical significance.

Results: Sixty-seven trials were included in the analysis. Significant relationships were seen between: $\mathrm{FEV}_{1}$ and any exacerbation (time to first exacerbation or patients with at least one exacerbation, $p=0.001$ ); between $\mathrm{FEV}_{1}$ and moderate-to-severe exacerbations (time to first exacerbation, patients with at least one exacerbation, or annualized rate, $p=0.045$ ); between $S G R Q$ score and any exacerbation (time to first exacerbation or patients with at least one exacerbation, $p=0.0002$ ) and between SGRQ score and moderate-to-severe exacerbations (time to first exacerbation or patients with at least one exacerbation, $p=0.0279$; annualized rate, $p=0.0024$ ). Relationships between $\mathrm{FEV}_{1}$ or SGRQ score and annualized exacerbation rate for any exacerbation or hospitalized exacerbations were not significant.

Conclusions: The regression analysis demonstrated a significant association between improvements in FEV $\mathrm{V}_{1}$ and SGRQ score and lower risk for COPD exacerbations. Even in cases of non-significant relationships, results were in the expected direction with few exceptions. The results of this analysis offer health care providers and payers a broader picture of the relationship between exacerbations and mean change in $\mathrm{FEV}_{1}$ as well as SGRQ score, and will help inform clinical and formulary-making decisions while stimulating new research questions for future prospective studies.
\end{abstract}

Keywords: COPD, Exacerbations, FEV 1 , SGRQ, Health resource utilization, Regression analysis

\footnotetext{
* Correspondence: amber.martin@evidera.com

${ }^{1}$ Evidera, 430 Bedford Street, Suite 300, 02420 Lexington, MA, USA

Full list of author information is available at the end of the article
} 


\section{Background}

Chronic obstructive pulmonary disease (COPD) is characterized by persistent airway obstruction related to chronic inflammatory responses in the lungs with symptoms including disabling dyspnea, fatigue, and persistent cough with excessive sputum. Exacerbations are characterized by a sustained acute worsening of respiratory symptoms beyond daily fluctuations, which leads to changes in medication use. Due to the disease symptoms, COPD patients often have a reduced capacity for physical activity and this may worsen potential systemic manifestations of the disease, such as cardiovascular and psychiatric comorbidities. The global prevalence of COPD is estimated to be $9.2 \%$ [1] with variable estimates, ranging from $3.9 \%$ [2] in the Netherlands to $20.9 \%$ in the US, [3] when reported by country. Therefore, COPD presents a major clinical and humanistic burden, [4] despite the availability and use of standard treatments, which aim to relieve symptoms and slow disease progression [5].

This heavy disease toll inevitably focuses interest on how patients are treated and the extent to which medications produce meaningful benefits. Assessment of such value in clinical trials has traditionally relied on measures of lung function (such as forced expiratory volume in one second $\left.\left[\mathrm{FEV}_{1}\right]\right)$, symptom control, health status, and rates of exacerbation over a period of up to one year. Exacerbations are a particularly important marker, not least because they are a key driver of health resource use (HRU), such as emergency department visits, antibiotic use and hospitalization. Evidence of this includes the fact that an exacerbation can cost upwards of $\$ 7,000$ each, depending on its severity and whether the patient is hospitalized [6]. Unsurprisingly, payers tend to focus on this outcome in their formulary considerations, with the expectation that decreased exacerbation rates will likely result in lower costs for their plan.

The clinical and economic importance of exacerbations in COPD invites questions about their interrelationship with other well-established measures of treatment effect. These include, for example, persistent and/or uncontrolled disease symptoms and health status as measured by the St. George's Respiratory Questionnaire [SGRQ] - which captures symptoms, impact on patient well-being, and activities of daily living. Additionally, clinically relevant improvements in lung function measures such as $\mathrm{FEV}_{1}$, are often required by regulators for certain drug approval processes. Of note, previous studies have looked at the link between $\mathrm{FEV}_{1}$ and SGRQ score $[7,8]$ but their relationship to longerterm outcomes, such as exacerbations and HRU, is not well-known and/or accepted, and this may account for why they have received comparatively less consideration from clinicians and payers.
Against this background, the current study aimed to investigate the relationship between changes in $\mathrm{FEV}_{1}$ and SGRQ score and economically significant outcomes of exacerbations and HRU, by conducting a systematic literature review (SLR) and regression analysis of relevant studies of pharmacological interventions for COPD. The results of this analysis will help the interpretation of clinical trial results and provide insights into whether or how the effects of COPD treatment seen in such studies relate to long-term clinical benefits.

\section{Methods \\ Literature review \\ Search strategy}

We systematically reviewed MEDLINE- (via PubMed), Embase-, and the Cochrane Central Register of Controlled Trials (CENTRAL) -indexed literature published from January 1, 2002 through October 1, 2014. The search algorithms used keywords for COPD paired with terms for the endpoints of interest-SGRQ, $\mathrm{FEV}_{1}$, exacerbations, and HRU. Limits included clinical trials on humans published in English.

\section{Study selection}

Following the literature search, all titles and abstracts identified from MEDLINE, Embase, and CENTRAL were manually reviewed against the inclusion and exclusion criteria using PICOS (Patient, Interventions, Comparisons, Outcomes, Study Design)-related elements. Studies were required to report on at least 20 adult COPD patients, to evaluate pharmacologic treatments labeled for or intended for use as treatment of COPD with any comparator treatment, to report mean change in either $\mathrm{FEV}_{1}$ or SGRQ score and either COPD exacerbations or any HRU endpoint, and to be a randomized controlled trial (RCT). A single investigator screened all abstracts identified through the searches, according to the specified inclusion and exclusion criteria. The fulltext articles of accepted studies that passed abstract screening were retrieved for further review. Screening was conducted by a single investigator using the same inclusion and exclusion criteria that had been applied at the abstract level. All excluded studies were confirmed by a second, senior investigator and any discrepancies between the two investigators were resolved by involvement of a third investigator.

\section{Data extraction process}

The results of all accepted studies identified as part of the SLR were extracted by a single investigator trained in the critical assessment of evidence, with validation performed by a senior investigator. Trial quality and risk of bias were assessed during extraction for each included study using the Jadad quality score assessment. 


\section{Statistical analysis}

The analyses relating measures of $\mathrm{FEV}_{1}$ and SGRQ total score to exacerbations and HRU followed the metaanalyses methods outlined by Johnson et al. [9] Each trial supplied one or more pairs of data points on the treatment effects of interest. These predictor/outcome pairs from each of the studies were analyzed using sample-size weighted regression analyses, which estimated a regression slope relating the two treatment effects, as well as a confidence interval and a test of statistical significance. In general, the predictor was a relative treatment effect for change in SGRQ or trough $\mathrm{FEV}_{1}$, and the outcome was a log-relative-risk or lograte for exacerbations. Pre-bronchodilator $\mathrm{FEV}_{1}$ was considered as equivalent to trough $\mathrm{FEV}_{1}$ for analysis, while post-bronchodilator measures and $\mathrm{FEV}_{1}$ that was unspecified were not included. Primary analyses were designed to avoid the use of an intercept in the regressions, but fit was superior with an intercept included.

For the analyses of patients experiencing at least one exacerbation, studies were included if they reported on exacerbations of all severities. For analyses of patients experiencing at least one moderate-to-severe exacerbation, studies were included if they reported on exacerbations that required antibiotics, oral corticosteroids (OCS), and/ or hospitalization. Data on time to first exacerbation or the number of patients with at least one exacerbation were combined for analysis. COPD exacerbations reported as an adverse event were not included in analysis. All studies reporting data at timepoints $\geq 24$ weeks were eligible for inclusion in the analyses. Separate analyses were conducted for all timepoints $\geq 24$ weeks and $\geq 48$ weeks.

\section{Results}

\section{Literature review}

The literature review identified 67 trials reporting endpoints of interest at timepoints $\geq 24$ weeks that were eligible for inclusion in the regression analysis. Fig. 1 outlines the overall search hits and study attrition during screening and analysis.

\section{Regression analysis}

In the figures representing the analyses, each point in the plot represents a study comparison for two effects. For instance, the point in the middle of Fig. 2 is from Bateman et al. [10] and represents their findings in the comparison of tiotropium $5 \mathrm{mg}$ (via the Respimat ${ }^{\circ}$ inhaler) vs. placebo. In this example, the difference between the two treatments in trough $\mathrm{FEV}_{1}$ change was -0.10 , and the hazard ratio (HR) for any exacerbation risk was 0.693 (for a $\log -\mathrm{HR}$ of -0.37). Each study with two arms (one treatment comparison, e.g. treatment A

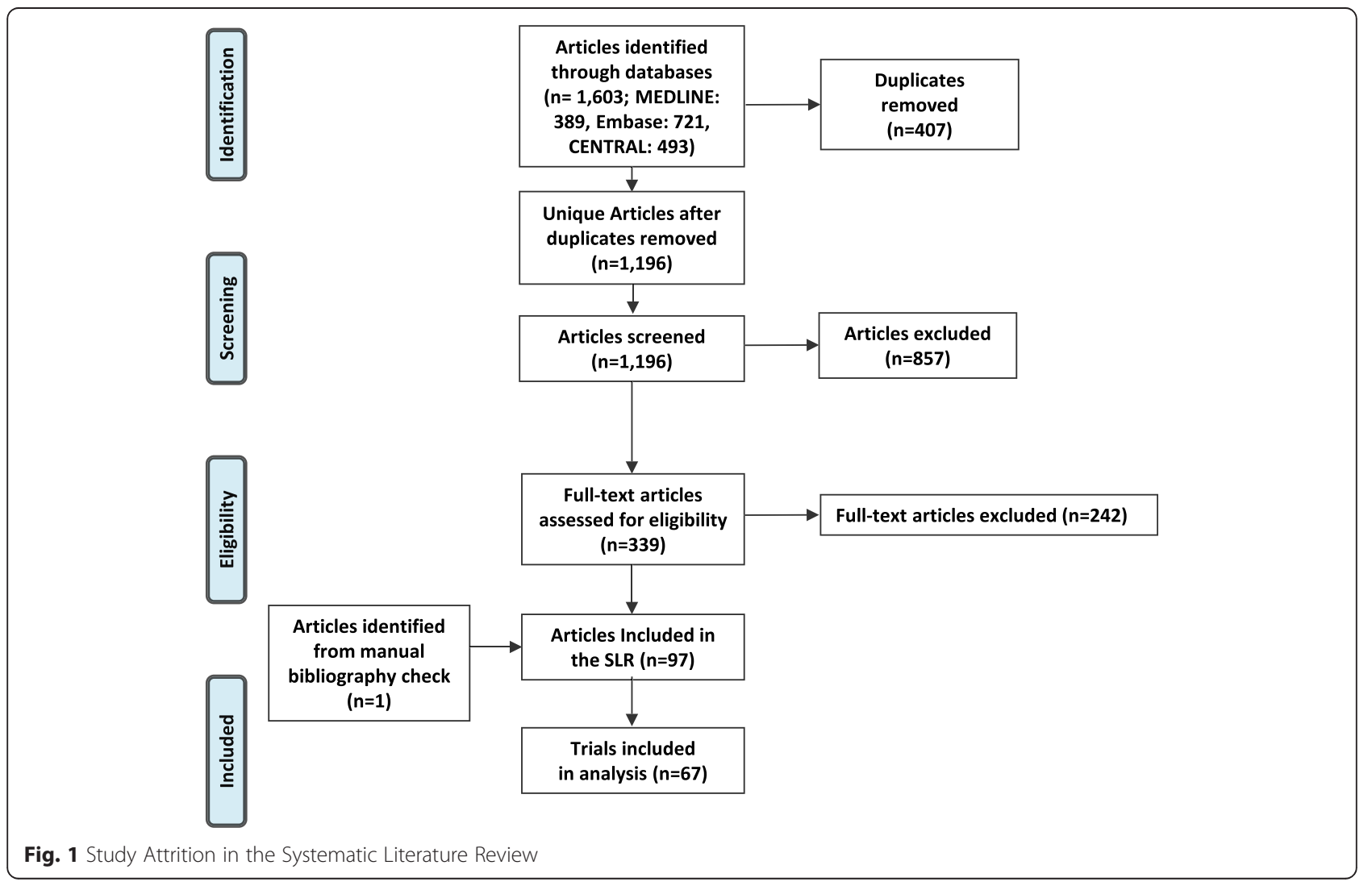




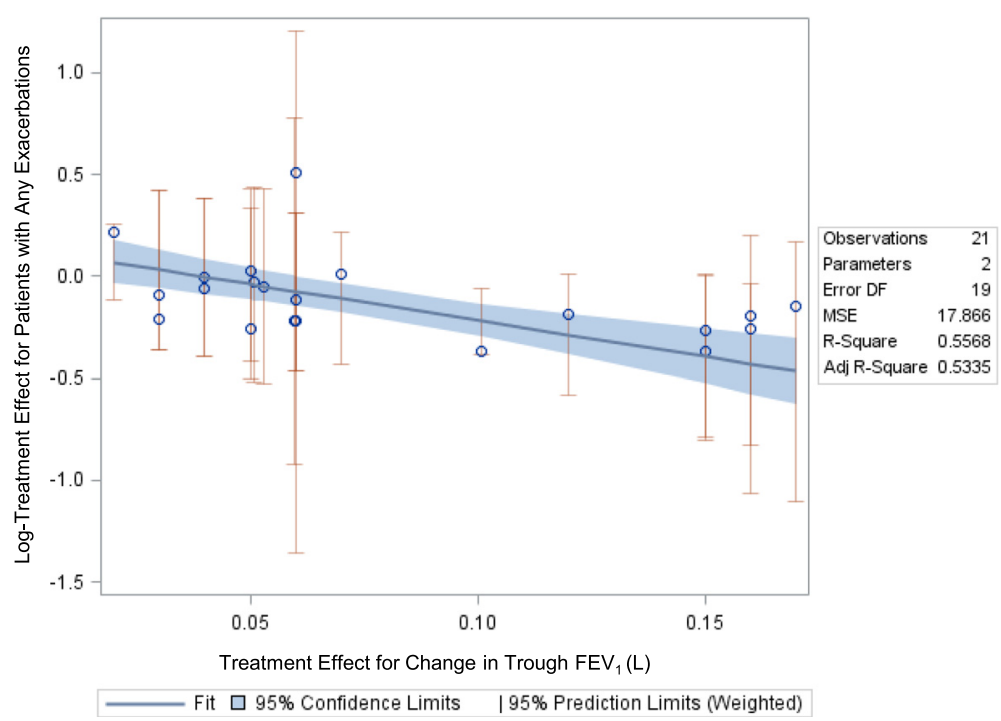

Fig. 2 Relationship between Mean Change in Trough FEV 1 and Relative Risk for Any Exacerbation

vs. treatment B) and with sufficient data contributed one data point to the analysis; studies with three arms (two treatment comparisons, e.g. A vs. B and A vs. C) contributed two data points.

Any given slope can be interpreted by determining what difference between treatments in log-exacerbation risk one would expect given the difference in trough $\mathrm{FEV}_{1}$ change. The predicted log-relative-risk of exacerbation in studies like Bateman 2010 is:

$$
\begin{aligned}
\ln (\text { RRAnyExacerbation }) & =\text { Intercept }+ \text { Slope } \\
& * \text { Difference in trough } \mathrm{FEV}_{1} \text { change } .
\end{aligned}
$$

Or

$$
\begin{aligned}
\ln (\text { RRAnyExacerbation }) & =0.14-3.56(0.10), \\
& =-0.22 .
\end{aligned}
$$

As $\exp (-0.22)=0.80$, we can predict that the relative risk of exacerbation in studies like Bateman 2010 will be $20 \%$ lower for active treatment than for control. As noted above and in the plot, in Bateman 2010 the relative risk of any exacerbation was actually slightly lower than this value (0.693).

\section{Relationships with exacerbations at $\geq \mathbf{4 8}$ weeks Forced Expiratory Volume in One Second (trough FEV ${ }_{1}$ )}

Mean Change in Trough FEV 1 and COPD Patients' Risk for Any Exacerbation The relationship between relative treatment effects on change in $\mathrm{FEV}_{1}$ and any exacerbation was of moderate strength and was statistically significant (slope: $-3.56, p=0.0001$; Fig. 2) when defining the exacerbation outcome as time to first exacerbation or the number of patients with at least one exacerbation. No relationship was found (slope: 0.078, $p=0.9199$ ) between treatment effects on $\mathrm{FEV}_{1}$ and annualized exacerbation rate. Figure 2 plots the relationship between the mean difference in trough $\mathrm{FEV}_{1}$ and relative risk for any exacerbation and Table 1 shows the raw trial data contributing to this analysis.

Mean Change in Trough $\mathrm{FEV}_{1}$ and COPD Patients' Risk for Moderate-to-Severe Exacerbations The relationship between relative treatment effects on change in $\mathrm{FEV}_{1}$ and moderate-to-severe exacerbations was of moderate strength and was statistically significant (slope: -1.46, $p=0.045$; Fig. 3) when defining the exacerbation outcome either as time to first exacerbation, the number of patients with at least one exacerbation, or as annualized exacerbation rates. Figure 3 shows the relationship between the mean difference in trough $\mathrm{FEV}_{1}$ and the relative risk for a moderate-to-severe exacerbation. Table 2 shows the raw trial data contributing to this analysis.

\section{St. George's respiratory questionnaire}

Mean Change in SGRQ Total Score and COPD Patients' Risk for Any Exacerbations The relationship between relative treatment effects for change in SGRQ score and any exacerbation was of moderate strength (slope: $0.112, p=0.0002$; Fig. 4 ) and was statistically significant when defining the exacerbation outcome as time to first-exacerbation or the number of patients with at least one exacerbation. The relationship was weaker and not statistically significant (slope: 0.014, $p=0.2825$ ) when examining annualized exacerbation rates. Figure 4 shows the relationship between the mean difference in SGRQ score and relative risk for any exacerbation and Table 3 shows the raw trial data contributing to this analysis. 
Table 1 Study Data for Trials Reporting Mean Change in Trough FEV 1 and Patients Experiencing Any Exacerbation

\begin{tabular}{|c|c|c|c|c|c|c|c|c|c|}
\hline $\begin{array}{l}\text { Author, } \\
\text { Year }\end{array}$ & Treatment & $\begin{array}{l}\text { Time } \\
\text { point } \\
\text { (weeks) }\end{array}$ & $\begin{array}{l}\mathrm{N} \\
\text { Randomized }\end{array}$ & $\begin{array}{l}\text { Definition of } \\
\text { exacerbation }\end{array}$ & $\begin{array}{l}\text { Annual } \\
\text { exacerbation } \\
\text { rate }\end{array}$ & $\begin{array}{l}\mathrm{N} \text { with any } \\
\text { exacerbation }\end{array}$ & $\begin{array}{l}\text { Comparison } \\
\text { data for Time } \\
\text { to first } \\
\text { exacerbation } \\
\text { (Hazard ratio) }\end{array}$ & $\begin{array}{l}\text { Mean } \\
\text { change } \\
\text { in } \\
\text { Trough } \\
\mathrm{FEV}_{1}(\mathrm{~L})\end{array}$ & $\begin{array}{l}\text { Comparison } \\
\text { data for } \\
\text { Trough FEV }{ }_{1} \\
\text { (treatment } \\
\text { difference) }\end{array}$ \\
\hline \multirow[t]{2}{*}{$\begin{array}{l}\text { Bateman, } \\
2010[10]\end{array}$} & Tiotropium 5 ug & 48 & 1989 & $\mathrm{~B}+$ & 0.12 & 685 & $\begin{array}{l}\text { Tio5 vs. } \\
\text { Placebo: } 0.69\end{array}$ & 0.119 & - \\
\hline & Placebo & 48 & 2002 & & 0.15 & 842 & & 0.018 & - \\
\hline \multirow[t]{3}{*}{$\begin{array}{l}\text { Calverley, } \\
2010[12]\end{array}$} & $\begin{array}{l}\text { Beclomethasone/formoterol } \\
\text { pMDI 400/24 } \mu \mathrm{g}\end{array}$ & 48 & 237 & NR & 0.074 & 64 & - & 0.077 & $\begin{array}{l}\text { B/F pMDI vs. } \\
\text { F-DPI: } 0.051\end{array}$ \\
\hline & $\begin{array}{l}\text { Budesonide/formoterol } \\
\text { DPI)800/24 } \mu \mathrm{g}\end{array}$ & 48 & 242 & & 0.033 & 64 & - & 0.08 & $\begin{array}{l}\text { B/F dry vs. F- } \\
\text { DPI: } 0.053\end{array}$ \\
\hline & Formoterol DPI $12 \mu \mathrm{g}$ & 48 & 239 & & 0.04 & 66 & - & 0.026 & - \\
\hline \multirow[t]{3}{*}{$\begin{array}{l}\text { Chapman, } \\
2011[13]\end{array}$} & Indacaterol, $150 \mu \mathrm{g}$ & 52 & 420 & A & - & - & $\begin{array}{l}\text { Ind150 vs. } \\
\text { Placebo: } 0.82\end{array}$ & 0.12 & - \\
\hline & Indacaterol, $300 \mu \mathrm{g}$ & 52 & 418 & & - & - & $\begin{array}{l}\text { Ind300 vs. } \\
\text { Placebo: } 0.86\end{array}$ & 0.13 & - \\
\hline & Placebo & 52 & 425 & & - & - & & -0.04 & - \\
\hline \multirow[t]{4}{*}{$\begin{array}{l}\text { Dahl, } 2010 \\
\text { [14] }\end{array}$} & Indacaterol $300 \mu \mathrm{g}$ & 52 & 437 & A & - & - & $\begin{array}{l}\text { Inda300 vs. } \\
\text { Placebo: } 0.77\end{array}$ & - & $\begin{array}{l}\text { Inda300 vs. } \\
\text { Placebo: } 0.16\end{array}$ \\
\hline & Indacaterol $600 \mu \mathrm{g}$ & 52 & 428 & & - & - & $\begin{array}{l}\text { Inda600 vs. } \\
\text { Placebo: } 0.69\end{array}$ & - & $\begin{array}{l}\text { Inda600 vs. } \\
\text { Placebo: } 0.15\end{array}$ \\
\hline & Formoterol & 52 & 435 & & - & - & $\begin{array}{l}\text { F vs. Placebo: } \\
0.77\end{array}$ & - & $\begin{array}{l}\text { F vs. } \\
\text { Placebo: } 0.05\end{array}$ \\
\hline & Placebo & 52 & 432 & & - & - & & - & - \\
\hline \multirow[t]{4}{*}{$\begin{array}{l}\text { Decramer, } \\
2013 \text { [15] }\end{array}$} & Tiotropium bromide $18 \mu \mathrm{g}$ & 26 & 1721 & C & - & - & $\begin{array}{l}\text { Tio18 vs. } \\
\text { Inda150: } 0.81\end{array}$ & - & $\begin{array}{l}\text { Tio18 vs. } \\
\text { Inda150: } 0.02\end{array}$ \\
\hline & $\begin{array}{l}\text { Indacaterol maleate } 150 \mu \mathrm{g} \\
\text { once-daily }\end{array}$ & 26 & 1723 & & - & & - & - & - \\
\hline & Tiotropium bromide $18 \mu \mathrm{g}$ & 52 & 1721 & & 0.07 & 547 & - & 0.092 & - \\
\hline & $\begin{array}{l}\text { Indacaterol maleate } 150 \mu \mathrm{g} \\
\text { once-daily }\end{array}$ & 52 & 1723 & & 0.1 & 619 & - & 0.073 & - \\
\hline \multirow[t]{2}{*}{$\begin{array}{l}\text { Dusser, } \\
2006[16]\end{array}$} & $\begin{array}{l}\text { Tiotropium } 18 \mu \mathrm{g} \\
\text { once daily }\end{array}$ & 48 & 500 & C & - & 248 & - & - & - \\
\hline & Placebo & 48 & 510 & & - & 305 & - & - & $\begin{array}{l}\text { Tio18 vs. } \\
\text { Placebo: } 0.12\end{array}$ \\
\hline \multirow[t]{2}{*}{$\begin{array}{l}\text { Ferguson, } \\
2008[17]\end{array}$} & $\begin{array}{l}\text { Fluticasone propionate/ } \\
\text { salmeterol (FSC) } 250 / 50\end{array}$ & 52 & 394 & C & - & 343 & - & -0.012 & - \\
\hline & Salmeterol $50 \mu \mathrm{g}$ & 52 & 388 & & - & 335 & - & -0.082 & - \\
\hline \multirow{2}{*}{$\begin{array}{l}\text { van } \\
\text { Grunsven, } \\
2003[18]\end{array}$} & $\begin{array}{l}\text { Fluticasone propionate } \\
\text { (Flixotides) } 250 \mu \mathrm{g} \text { bid }\end{array}$ & 103 & 24 & $\mathrm{D}$ & - & 5 & - & -0.12 & $\begin{array}{l}\text { F250 vs. } \\
\text { Placebo: } 0.06\end{array}$ \\
\hline & Placebo bid & 103 & 24 & & - & 3 & - & -0.17 & - \\
\hline \multirow[t]{2}{*}{$\begin{array}{l}\text { Vincken, } \\
2002[19]\end{array}$} & $\begin{array}{l}\text { Tiotropium } 18 \mu \mathrm{g} \\
\mathrm{qd} \text { in the morning }\end{array}$ & 52 & 356 & $\mathrm{~B}$ & - & 125 & - & 0.12 & - \\
\hline & Ipratropium 40 mg qid & 52 & 179 & & - & 82 & - & -0.03 & - \\
\hline \multirow[t]{2}{*}{$\begin{array}{l}\text { Wouters, } \\
2005[20]\end{array}$} & $\begin{array}{l}\text { Salmeterol/fluticasone } \\
(3 \text { month run in period of } \\
\text { salmeterol } 50 \mu \mathrm{g} \text { and } \\
\text { fluticasone } 500 \mu \mathrm{g} \text { bid })\end{array}$ & 52 & 189 & $\mathrm{E}$ & - & 115 & - & -0.04 & $\begin{array}{l}\text { S/F vS. S: } \\
0.05\end{array}$ \\
\hline & $\begin{array}{l}\text { Salmeterol (3 month run in } \\
\text { period of salmeterol } 50 \mu \mathrm{g} \\
\text { and fluticasone } 500 \mu \mathrm{g} \mathrm{bid})\end{array}$ & 52 & 184 & & - & 109 & - & -0.1 & - \\
\hline \multirow{2}{*}{$\begin{array}{l}\text { Zhou, } \\
2006 \text { [21] }\end{array}$} & Theophylline & 52 & 57 & C & - & 26 & - & 0.0063 & - \\
\hline & Placebo & 52 & 53 & & - & 30 & - & -0.0533 & - \\
\hline
\end{tabular}


Table 1 Study Data for Trials Reporting Mean Change in Trough FEV 1 and Patients Experiencing Any Exacerbation (Continued)

\begin{tabular}{|c|c|c|c|c|c|c|c|c|c|}
\hline \multirow{8}{*}{$\begin{array}{l}\text { Dransfield, } \\
2013 \text { [22] }\end{array}$} & Vilanterol $25 \mu \mathrm{g}$ & 52 & 409 & \multirow[t]{8}{*}{ A } & - & 203 & - & -0.04 & - \\
\hline & $\begin{array}{l}\text { Fluticasone furoate } 50 \mu \mathrm{g}+ \\
\text { Vilanterol } 25 \mu \mathrm{g}\end{array}$ & 52 & 408 & & - & 190 & - & 0 & - \\
\hline & $\begin{array}{l}\text { Fluticasone furoate } 100 \mu \mathrm{g} \\
+ \text { Vilanterol } 25 \mu \mathrm{g}\end{array}$ & 52 & 403 & & - & 161 & - & 0.02 & - \\
\hline & $\begin{array}{l}\text { Fluticasone furoate } 200 \mu \mathrm{g} \\
\text { + Vilanterol } 25 \mu \mathrm{g}\end{array}$ & 52 & 402 & & - & 178 & - & 0.02 & - \\
\hline & Vilanterol $25 \mu \mathrm{g}$ & 52 & 409 & & - & 197 & - & -0.02 & - \\
\hline & $\begin{array}{l}\text { Fluticasone furoate } 50 \mu \mathrm{g}+ \\
\text { Vilanterol } 25 \mu \mathrm{g}\end{array}$ & 52 & 412 & & - & 198 & - & 0.02 & - \\
\hline & $\begin{array}{l}\text { Fluticasone furoate } 100 \mu \mathrm{g} \\
\text { + Vilanterol } 25 \mu \mathrm{g}\end{array}$ & 52 & 403 & & - & 177 & - & 0.01 & - \\
\hline & $\begin{array}{l}\text { Fluticasone furoate } 200 \mu \mathrm{g} \\
+ \text { Vilanterol } 25 \mu \mathrm{g}\end{array}$ & 52 & 409 & & - & 160 & - & 0.01 & - \\
\hline
\end{tabular}

\section{Exacerbation Definitions:}

A:Symptom deterioration requiring antibiotics, systemic corticosteroids, and/or hospitalization

$B: A$ complex of respiratory events lasting $\geq 3$ days

$B+: A$ complex of respiratory events lasting $\geq 3$ days requiring treatment

C:Worsening of at least two symptoms for at least two days

D:Having two of the following three symptoms: increased cough, wheezing and/or dyspnea; change in sputum color; use of bronchodilator rescue medication

E:If a patient has in $\geq 2$ consecutive days used $\geq 3$ extra inhalations of salbutamol per 24 hours above their reference rescue value

- = Not Reported

Mean Change in SGRQ Total Score and COPD Patients' Risk for Moderate-to-severe Exacerbations The relationship between relative treatment effects for change in SGRQ score and a moderate-to-severe exacerbation was of moderate strength and was statistically significant when defining the exacerbation outcome as either the number of patients with at least one exacerbation (slope: $0.046, p=0.0279$, Fig. 5) or as an annualized exacerbation rate (slope: 0.056, $p=0.0024$, figure not shown). Figure 5 shows the relationship between the mean difference in SGRQ score and the relative risk for a moderate-to-severe exacerbation and Table 4 shows the raw trial data contributing to this analysis.

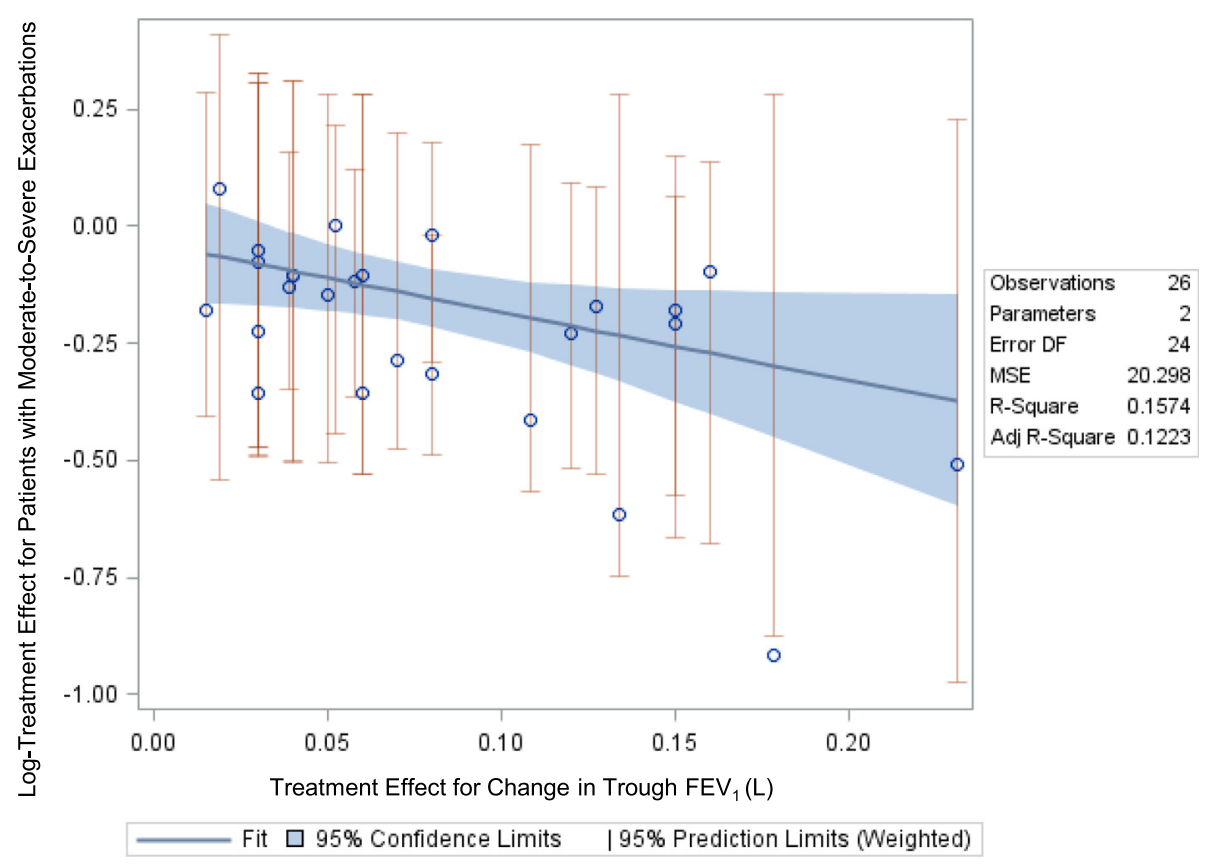

Fig. 3 Relationship between Mean Change in Trough FEV 1 and Risk for a Moderate-to-Severe Exacerbation 
Table 2 Study Data for Trials Reporting Mean change in FEV ${ }_{1}$ and Patients Experiencing Moderate-to-Severe COPD Exacerbation

\begin{tabular}{|c|c|c|c|c|c|c|c|c|}
\hline Author, Year & Treatment & $\begin{array}{l}\text { Time } \\
\text { point } \\
\text { (weeks) }\end{array}$ & N Randomized & $\begin{array}{l}\text { Annual } \\
\text { exacerbation } \\
\text { rate }(M-S)\end{array}$ & $\begin{array}{l}\mathrm{N} \text { with M-S } \\
\text { exacerbation }\end{array}$ & $\begin{array}{l}\text { Comparison } \\
\text { data for } \\
\text { Time to first } \\
\text { exacerbation } \\
\text { (Hazard ratio) }\end{array}$ & $\begin{array}{l}\text { Mean } \\
\text { change } \\
\text { in Trough } \\
\mathrm{FEV}_{1}(\mathrm{~L})\end{array}$ & $\begin{array}{l}\text { Comparison } \\
\text { data for Trough } \\
\mathrm{FEV}_{1} \\
\text { (treatment } \\
\text { difference) }\end{array}$ \\
\hline \multirow[t]{2}{*}{$\begin{array}{l}\text { Anzueto, } \\
2009[23]\end{array}$} & $\begin{array}{l}\text { Fluticasone propionate/ } \\
\text { salmeterol } 250 \mathrm{mcg} / 50 \\
\text { mcg bid }\end{array}$ & 52 & 394 & 1.1 & 208 & $\begin{array}{l}\text { FP250 + S50 vs. } \\
\text { S50: } 0.73\end{array}$ & -0.017 & - \\
\hline & Salmeterol $50 \mathrm{mcg}$ bid & 52 & 403 & 1.59 & 234 & - & -0.097 & - \\
\hline \multirow[t]{3}{*}{$\begin{array}{l}\text { Bateman, } \\
2010[10]\end{array}$} & $\begin{array}{l}\text { Tiotropium } 5 \mu \mathrm{g} \text { orally } \\
\text { inhaled once daily }\end{array}$ & 48 & 670 & 0.93 & 249 & - & 0.08 & $\begin{array}{l}\text { Tio5 vs. } \\
\text { Placebo: } 0.127\end{array}$ \\
\hline & $\begin{array}{l}\text { Tiotropium } 10 \mu \mathrm{g} \text { orally } \\
\text { inhaled once daily }\end{array}$ & 48 & 667 & 1.02 & 246 & - & 0.11 & $\begin{array}{l}\text { Tio10 vs. } \\
\text { Placebo: } 0.150\end{array}$ \\
\hline & Placebo & 48 & 653 & 1.91 & 288 & - & -0.04 & \\
\hline \multirow[t]{4}{*}{$\begin{array}{l}\text { Dahl, } 2010 \\
\text { [14] }\end{array}$} & Indacaterol $300 \mu \mathrm{g}$ & 52 & 437 & 0.6 & 133 & - & - & $\begin{array}{l}\text { Inda300 vs. } \\
\text { Placebo: } 0.16\end{array}$ \\
\hline & Indacaterol $600 \mu \mathrm{g}$ & 52 & 428 & 0.57 & 116 & - & - & $\begin{array}{l}\text { Inda600 vs. } \\
\text { Placebo: } 0.15\end{array}$ \\
\hline & Formoterol & 52 & 435 & 0.56 & 126 & - & - & F vs. Placebo: 0.05 \\
\hline & Placebo & 52 & 432 & 0.74 & 145 & - & - & \\
\hline \multirow[t]{3}{*}{$\begin{array}{l}\text { Donohue, } \\
2014 \text { [24] }\end{array}$} & UMEC/NI 125/25 mcg & 52 & 226 & - & 30 & $\begin{array}{l}\text { UMECNI vs. } \\
\text { Placebo: } 0.6\end{array}$ & 0.18 & $\begin{array}{l}\text { UMECNI vs. } \\
\text { Placebo: } 0.231\end{array}$ \\
\hline & UMEC 125 mcg & 52 & 227 & - & 34 & $\begin{array}{l}\text { UMEC vs. } \\
\text { Placebo: } 0.4\end{array}$ & 0.13 & $\begin{array}{l}\text { UMEC vs. } \\
\text { Placebo: } 0.178\end{array}$ \\
\hline & Placebo & 52 & 109 & - & 26 & - & -0.05 & - \\
\hline \multirow[t]{2}{*}{$\begin{array}{l}\text { Ferguson, } \\
2008[17]\end{array}$} & $\begin{array}{l}\text { Fluticasone propionate/ } \\
\text { salmeterol (FSC) } 250 / 50\end{array}$ & 52 & 394 & 1.06 & 211 & FP/S vs. S: 0.75 & -0.012 & - \\
\hline & Salmeterol $50 \mu \mathrm{g}$ & 52 & 388 & 1.53 & 230 & - & -0.082 & - \\
\hline \multirow[t]{3}{*}{$\begin{array}{l}\text { Kerwin, } \\
2012[25]\end{array}$} & NVA237 $50 \mu \mathrm{g}$ qd & 52 & 529 & 0.54 & & $\begin{array}{l}\text { NVA vs. } \\
\text { Placebo: } 0.66\end{array}$ & 0.112 & $\begin{array}{l}\text { NVA vs. } \\
\text { Placebo: } 0.108\end{array}$ \\
\hline & Tiotropium $18 \mu \mathrm{g}$ qd & 52 & 268 & - & - & NVA vs. Tio: 1.1 & 0.092 & NVA vs. Tio: 0.019 \\
\hline & Placebo & 52 & 269 & 0.8 & - & - & -0.097 & \\
\hline \multirow[t]{3}{*}{$\begin{array}{l}\text { Sharafkhaneh, } \\
2012 \text { [26] }\end{array}$} & $\begin{array}{l}\text { Budesonide/formoterol } \\
\text { pMDI } 160 / 4.5 \mu \mathrm{g} \times 2 \\
\text { inhalations bid }(320 / 9 \mu \mathrm{g})\end{array}$ & 52 & 407 & 0.867 & 169 & - & 0.07 & - \\
\hline & $\begin{array}{l}\text { Budesonide/formoterol } \\
\text { pMDI 80/4.5 } \mu \mathrm{g} \times 2 \\
\text { inhalations bid }(160 / 9 \mu \mathrm{g})\end{array}$ & 52 & 408 & 0.952 & 173 & - & 0.07 & - \\
\hline & $\begin{array}{l}\text { Formoterol DPI } 4.5 \mu \mathrm{g} \times 2 \\
\text { inhalations bid }(9 \mu \mathrm{g})\end{array}$ & 52 & 404 & 1.171 & 182 & - & 0.04 & - \\
\hline \multirow[t]{2}{*}{$\begin{array}{l}\text { Tang, } 2013 \\
{[27]}\end{array}$} & $\begin{array}{l}\text { Tiotropium } 5 \mu \mathrm{g} \\
(2 \times 2.5 \mu \mathrm{g} / \text { puff })\end{array}$ & 48 & 167 & - & 58 & $\begin{array}{l}\text { Tio5 vs. } \\
\text { Placebo: } 0.54\end{array}$ & - & $\begin{array}{l}\text { Tio5 vs. } \\
\text { Placebo: } 0.134\end{array}$ \\
\hline & Placebo (2 puffs) & 48 & 171 & - & 83 & - & - & - \\
\hline \multirow[t]{2}{*}{$\begin{array}{l}\text { Tashkin, } 2008 \\
\text { [11] }\end{array}$} & $\begin{array}{l}\text { Tiotropium } 18 \mu \mathrm{g} \text { once } \\
\text { daily; followed by } 40 \mu \mathrm{g} \\
\text { of ipratropium four times } \\
\text { daily for } 30 \text { days after } \\
4 \text { years of treatment. }\end{array}$ & 206 & 2987 & - & 2001 & - & 0.03 & - \\
\hline & $\begin{array}{l}\text { Placebo once daily; } \\
\text { followed by } 40 \mu \mathrm{g} \text { of } \\
\text { ipratropium four times } \\
\text { daily for } 30 \text { days after } \\
4 \text { years of treatment. }\end{array}$ & 206 & 3006 & - & 2049 & - & -0.05 & - \\
\hline $\begin{array}{l}\text { Calverley, } \\
2009[28]\end{array}$ & $\begin{array}{l}\text { Roflumilast } 500 \text { mcg } \\
\text { once per day }\end{array}$ & 52 & 765 & 1.08 & 344 & $\begin{array}{l}\text { ROLF500 vs. } \\
\text { Placebo } \\
\text { (Trial 1): } 0.88\end{array}$ & 0.046 & $\begin{array}{l}\text { ROLF500 vs. } \\
\text { Placebo } \\
\text { (Trial 1): } 0.039\end{array}$ \\
\hline
\end{tabular}


Table 2 Study Data for Trials Reporting Mean change in FEV 1 and Patients Experiencing Moderate-to-Severe COPD Exacerbation (Continued)

\begin{tabular}{|c|c|c|c|c|c|c|c|c|}
\hline & Placebo & 52 & 758 & 1.27 & 389 & - & 0.008 & - \\
\hline & $\begin{array}{l}\text { Roflumilast } 500 \mathrm{mcg} \text { once } \\
\text { per day }\end{array}$ & 52 & 772 & 1.21 & 373 & $\begin{array}{l}\text { ROLF500 vs. } \\
\text { Placebo } \\
\text { (Trial 2): } 0.89\end{array}$ & 0.033 & $\begin{array}{l}\text { ROLF500 vs. } \\
\text { Placebo } \\
\text { (Trial 2): } 0.058\end{array}$ \\
\hline & Placebo & 52 & 796 & 1.49 & 432 & - & -0.025 & - \\
\hline \multirow[t]{8}{*}{$\begin{array}{l}\text { Dransfield, } \\
2013 \text { [22] }\end{array}$} & Vilanterol $25 \mu \mathrm{g}$ & 52 & 409 & 1.05 & - & $\begin{array}{l}\text { FF } 200+V \text { vs. } \\
\text { V: } 0.9\end{array}$ & -0.04 & - \\
\hline & $\begin{array}{l}\text { Fluticasone furoate } 50 \mu \mathrm{g} \\
+ \text { Vilanterol } 25 \mu \mathrm{g}\end{array}$ & 52 & 408 & 0.92 & - & $\begin{array}{l}\text { FF100+V vs. } \\
\text { V: } 0.7\end{array}$ & 0 & - \\
\hline & $\begin{array}{l}\text { Fluticasone furoate } \\
100 \mu \mathrm{g}+\text { Vilanterol } 25 \mu \mathrm{g}\end{array}$ & 52 & 403 & 0.7 & - & $\begin{array}{l}\text { FF50 }+ \text { V vs. } \\
\text { V: } 0.9\end{array}$ & 0.02 & - \\
\hline & $\begin{array}{l}\text { Fluticasone furoate } \\
200 \mu \mathrm{g}+\text { Vilanterol } 25 \mu \mathrm{g}\end{array}$ & 52 & 402 & 0.9 & - & - & 0.02 & - \\
\hline & Vilanterol $25 \mu \mathrm{g}$ & 52 & 409 & 1.14 & - & $\begin{array}{l}F F 200+V \text { vs. } \\
\text { V: } 0.7\end{array}$ & -0.02 & - \\
\hline & $\begin{array}{l}\text { Fluticasone furoate } 50 \mu \mathrm{g} \\
+ \text { Vilanterol } 25 \mu \mathrm{g}\end{array}$ & 52 & 412 & 0.92 & - & $\begin{array}{l}\text { FF100+V vs. } \\
\text { V: } 0.8\end{array}$ & 0.02 & - \\
\hline & $\begin{array}{l}\text { Fluticasone furoate } \\
100 \mu \mathrm{g}+\text { Vilanterol } 25 \mu \mathrm{g}\end{array}$ & 52 & 403 & 0.9 & - & $\begin{array}{l}\text { FF50 + V vs. } \\
\text { V: } 0.9\end{array}$ & 0.01 & - \\
\hline & $\begin{array}{l}\text { Fluticasone furoate } \\
200 \mu \mathrm{g}+\text { Vilanterol } 25 \mu \mathrm{g}\end{array}$ & 52 & 409 & 0.79 & - & - & 0.01 & - \\
\hline \multirow[t]{4}{*}{$\begin{array}{l}\text { Jones, } \\
2011[29]\end{array}$} & Aclidinium 200 mg & 52 & 627 & & 167 & $\begin{array}{l}\text { Aclid200 vs. } \\
\text { Placebo } \\
\text { (Trial 1): } 0.00\end{array}$ & -0.013 & \\
\hline & Placebo & 52 & 216 & 0.46 & 55 & - & -0.065 & - \\
\hline & Aclidinium 200 mg & 52 & 600 & & 199 & - & -0.009 & - \\
\hline & Placebo & 52 & 204 & 0.8 & 81 & - & -0.024 & - \\
\hline
\end{tabular}

$\mathrm{M}-\mathrm{S}=$ moderate-to-severe

$-=$ Not reported

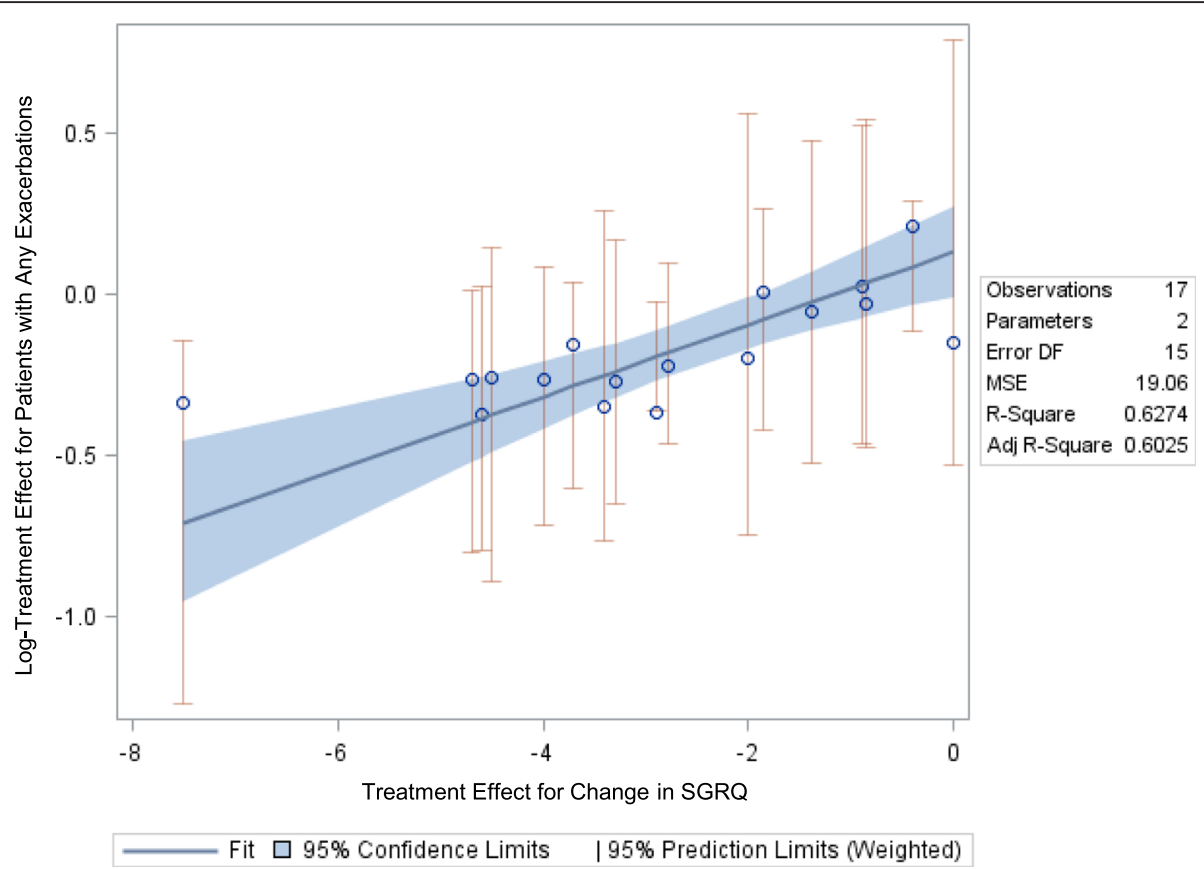

Fig. 4 Relationship between Mean Change in SGRQ Total Score and Risk for Any Exacerbation 
Table 3 Study Data for Trials Reporting Mean change in SGRQ Total Score and Patients Experiencing Any COPD Exacerbation

\begin{tabular}{|c|c|c|c|c|c|c|c|c|c|}
\hline $\begin{array}{l}\text { Author, } \\
\text { year }\end{array}$ & Treatment & $\begin{array}{l}\text { Time } \\
\text { point } \\
\text { (weeks) }\end{array}$ & $\begin{array}{l}\mathrm{N} \\
\text { Randomized }\end{array}$ & $\begin{array}{l}\text { Definition of } \\
\text { exacerbation }\end{array}$ & $\begin{array}{l}\text { Annual } \\
\text { exacerbation } \\
\text { rate (any) }\end{array}$ & $\begin{array}{l}\mathrm{N} \text { with any } \\
\text { exacerbation }\end{array}$ & $\begin{array}{l}\text { Comparison } \\
\text { data for } \\
\text { Time to first } \\
\text { exacerbation } \\
\text { (Hazard ratio) }\end{array}$ & $\begin{array}{l}\text { Mean } \\
\text { change } \\
\text { in SGRQ } \\
\text { Total Score }\end{array}$ & $\begin{array}{l}\text { Comparison } \\
\text { data for SGRQ } \\
\text { (treatment } \\
\text { difference) }\end{array}$ \\
\hline \multirow[t]{2}{*}{$\begin{array}{l}\text { Bateman, } \\
2010 \text { [10] }\end{array}$} & Tiotropium $5 \mu \mathrm{g}$ & 48 & 1989 & $\mathrm{~B}+$ & 0.69 & 685 & $\begin{array}{l}\text { Tio vs. } \\
\text { placebo: } 0.93\end{array}$ & -4.7 & $\begin{array}{l}\text { Tio5 vs. } \\
\text { placebo: }-2.9\end{array}$ \\
\hline & Placebo & 48 & 2002 & & 0.87 & 842 & & -1.8 & - \\
\hline \multirow[t]{4}{*}{$\begin{array}{l}\text { Calverley, } \\
2003[30]\end{array}$} & $\begin{array}{l}\text { Budesonide/formoterol } \\
320 / 9 \text { mg (bid) }\end{array}$ & 52 & 254 & A & 1.38 & - & $\begin{array}{l}B+F \text { vs. } \\
\text { B: } 0.77\end{array}$ & - & B +F vs. B: -4.5 \\
\hline & $\begin{array}{l}\text { Budesonide } 400 \mathrm{mg} \\
\text { (bid) }\end{array}$ & 52 & 257 & & 1.6 & - & $\begin{array}{l}B+F \text { vs. } \\
F: 0.71\end{array}$ & - & $B+F$ vs. F: -3.4 \\
\hline & Formoterol 9 mg (bid) & 52 & 255 & & 1.85 & - & $\begin{array}{l}\text { B + F vs. } \\
\text { Placebo: } 0.72\end{array}$ & - & $\begin{array}{l}\text { B +F vs. } \\
\text { Placebo: }-7.5\end{array}$ \\
\hline & Placebo & 52 & 256 & & 1.8 & - & - & - & - \\
\hline \multirow[t]{3}{*}{$\begin{array}{l}\text { Calverley, } \\
2010[12]\end{array}$} & $\begin{array}{l}\text { Beclomethasone/ } \\
\text { formoterol pMDI } \\
400 / 24 \mu \mathrm{g}\end{array}$ & 48 & 237 & NR & 0.414 & 64 & - & -3.75 & - \\
\hline & $\begin{array}{l}\text { Budesonide/formoterol } \\
\text { DPI 800/24 } \mu \mathrm{g}\end{array}$ & 48 & 242 & & 0.423 & 64 & - & -4.28 & - \\
\hline & Formoterol DPI $12 \mu \mathrm{g}$ & 48 & 239 & & 0.431 & 66 & - & -2.9 & - \\
\hline \multirow{2}{*}{$\begin{array}{l}\text { Casaburi, } \\
2002[31]\end{array}$} & Tiotropium $18 \mu \mathrm{g}$ & 52 & 550 & B & 0.76 & 198 & - & -3.2 & - \\
\hline & Placebo & 52 & 371 & & 0.95 & 156 & - & 0.5 & - \\
\hline \multirow[t]{3}{*}{$\begin{array}{l}\text { Chapman, } \\
2011[13]\end{array}$} & Indacaterol, $150 \mu \mathrm{g}$ & 52 & 420 & A & - & - & $\begin{array}{l}\text { Ind150 vs. } \\
\text { Placebo: } 0.82\end{array}$ & -7.5 & - \\
\hline & Indacaterol, 300 mg & 52 & 418 & & - & - & $\begin{array}{l}\text { Ind300 vs. } \\
\text { Placebo: } 0.86\end{array}$ & -5.5 & - \\
\hline & Placebo & 52 & 425 & & - & - & - & -5.5 & - \\
\hline \multirow[t]{4}{*}{$\begin{array}{l}\text { Dahl, } 2010 \\
\text { [14] }\end{array}$} & Indacaterol 300 mg & 52 & 437 & A & - & - & $\begin{array}{l}\text { Inda300 vs. } \\
\text { Placebo: } 0.77\end{array}$ & -6.5 & $\begin{array}{l}\text { Inda300 vs. } \\
\text { Placebo: }-4.7\end{array}$ \\
\hline & Indacaterol $600 \mu \mathrm{g}$ & 52 & 428 & & - & - & $\begin{array}{l}\text { Inda600 vs. } \\
\text { Placebo: } 0.69\end{array}$ & -7.2 & $\begin{array}{l}\text { Inda600 vs. } \\
\text { Placebo: }-4.6\end{array}$ \\
\hline & Formoterol & 52 & 435 & & - & - & $\begin{array}{l}\text { F vs. } \\
\text { Placebo: } 0.77\end{array}$ & -7 & F vs. Placebo: -4 \\
\hline & Placebo & 52 & 432 & & - & - & - & -1.7 & - \\
\hline \multirow[t]{4}{*}{$\begin{array}{l}\text { Decramer, } \\
2013[15]\end{array}$} & $\begin{array}{l}\text { Tiotropium bromide } \\
18 \mu \mathrm{g}\end{array}$ & 26 & 1721 & C & - & - & - & -5.2 & - \\
\hline & $\begin{array}{l}\text { Indacaterol maleate } \\
150 \text { mg once-daily }\end{array}$ & 26 & 1723 & & - & - & - & -4.5 & - \\
\hline & $\begin{array}{l}\text { Tiotropium bromide } \\
18 \mu \mathrm{g}\end{array}$ & 52 & 1721 & & 0.61 & 547 & - & -4.9 & - \\
\hline & $\begin{array}{l}\text { Indacaterol maleate } \\
150 \mu \mathrm{g} \text { once-daily }\end{array}$ & 52 & 1723 & & 0.79 & 619 & - & -4.5 & - \\
\hline \multirow[t]{2}{*}{$\begin{array}{l}\text { Ferguson, } \\
2008[17]\end{array}$} & $\begin{array}{l}\text { Fluticasone propionate/ } \\
\text { salmeterol (FSC) 250/50 }\end{array}$ & 52 & 394 & C & 4.82 & 343 & - & -3.49 & FP/S vs. S: -1.86 \\
\hline & Salmeterol $50 \mu \mathrm{g}$ & 52 & 388 & & 5.78 & 335 & - & -1.86 & - \\
\hline \multirow[t]{2}{*}{$\begin{array}{l}\text { Vincken, } \\
2002[19]\end{array}$} & $\begin{array}{l}\text { Tiotropium } 18 \mu \mathrm{g} \text { qd in } \\
\text { the morning }\end{array}$ & 52 & 356 & B & 0.73 & 125 & - & -3.74 & $\begin{array}{l}\text { Tio18 vs. } \\
\text { Ipra40: }-3.3\end{array}$ \\
\hline & Ipratropium $40 \mu \mathrm{g}$ qid & 52 & 179 & & 0.96 & 82 & - & -0.44 & - \\
\hline
\end{tabular}


Table 3 Study Data for Trials Reporting Mean change in SGRQ Total Score and Patients Experiencing Any COPD Exacerbation (Continued)

\begin{tabular}{|c|c|c|c|c|c|c|c|c|c|}
\hline \multirow[t]{2}{*}{$\begin{array}{l}\text { Wedzicha, } \\
2014 \text { [32] }\end{array}$} & $\begin{array}{l}\text { beclomethasone } \\
\text { dipropionate/formoterol } \\
\text { fumarate (BDP/FOR) 100/ } \\
6 \mu \mathrm{g}, 2 \text { inhalations BID }\end{array}$ & 48 & 602 & $\mathrm{~F}$ & 0.8 & 264 & $\begin{array}{l}\text { BDP + F vs. } \\
F: 0.8\end{array}$ & -3.55 & $\begin{array}{l}\text { BDP/F vs. } \\
\text { F: }-2.78\end{array}$ \\
\hline & $\begin{array}{l}\text { Formoterol fumarate } \\
\text { (FOR) } 12 \mu \mathrm{g}, 1 \\
\text { inhalation BID }\end{array}$ & 48 & 597 & & 1.12 & 294 & - & -0.77 & - \\
\hline \multirow[t]{2}{*}{$\begin{array}{l}\text { Wouters, } \\
2005 \text { [20] }\end{array}$} & $\begin{array}{l}\text { Salmeterol/fluticasone } \\
\text { ( } 3 \text { month run in period } \\
\text { of salmeterol } 50 \mu \mathrm{g} \text { and } \\
\text { fluticasone } 500 \mu \mathrm{g} \text { bid) }\end{array}$ & 52 & 189 & $E$ & - & 115 & - & 2.4 & S/F vs. S: -0.89 \\
\hline & $\begin{array}{l}\text { Salmeterol ( } 3 \text { month } \\
\text { run in period of } \\
\text { salmeterol } 50 \mu \mathrm{g} \text { and } \\
\text { fluticasone } 500 \mu \mathrm{g} \text { bid) }\end{array}$ & 52 & 184 & & - & 109 & - & 3.2 & - \\
\hline
\end{tabular}

\section{Exacerbation Definitions:}

A:Symptom deterioration requiring antibiotics, systemic corticosteroids, and/or hospitalization

$B: A$ complex of respiratory events lasting $\geq 3$ days

$B+: A$ complex of respiratory events lasting $\geq 3$ days requiring treatment

C:Worsening of at least two symptoms for at least two days

E:If a patient has in $\geq 2$ consecutive days used $\geq 3$ extra inhalations of salbutamol per 24 hours above their reference rescue value

F:An acute event characterized by a worsening of the patient's respiratory symptoms that is beyond normal day-to-day variations and leads to a change in medication

$-=$ Not reported

\section{Relationship between FEV 1 and SGRQ and Hospitalized COPD Exacerbations}

There were insufficient data to analyze association with allcause hospitalizations, and the annualized and patient-level data were combined for the analysis of hospitalizations due to exacerbations. Additionally, relative effects for the number of patients with an exacerbation were combined with annualized exacerbation rates to facilitate analyses.
FEV $_{1}$ and SGRQ

For both SGRQ score and $\mathrm{FEV}_{1}$, the plots indicate a somewhat weaker relationship with exacerbations resulting in hospitalization (compared to the findings for exacerbations overall). Results were not statistically significant $\left(\mathrm{FEV}_{1}\right.$ slope: $-1.49, p$-value $=0.174$ [Fig. 6]; SGRQ slope: 0.0518, $p=0.126$ [Fig. 7]) for either relationship.

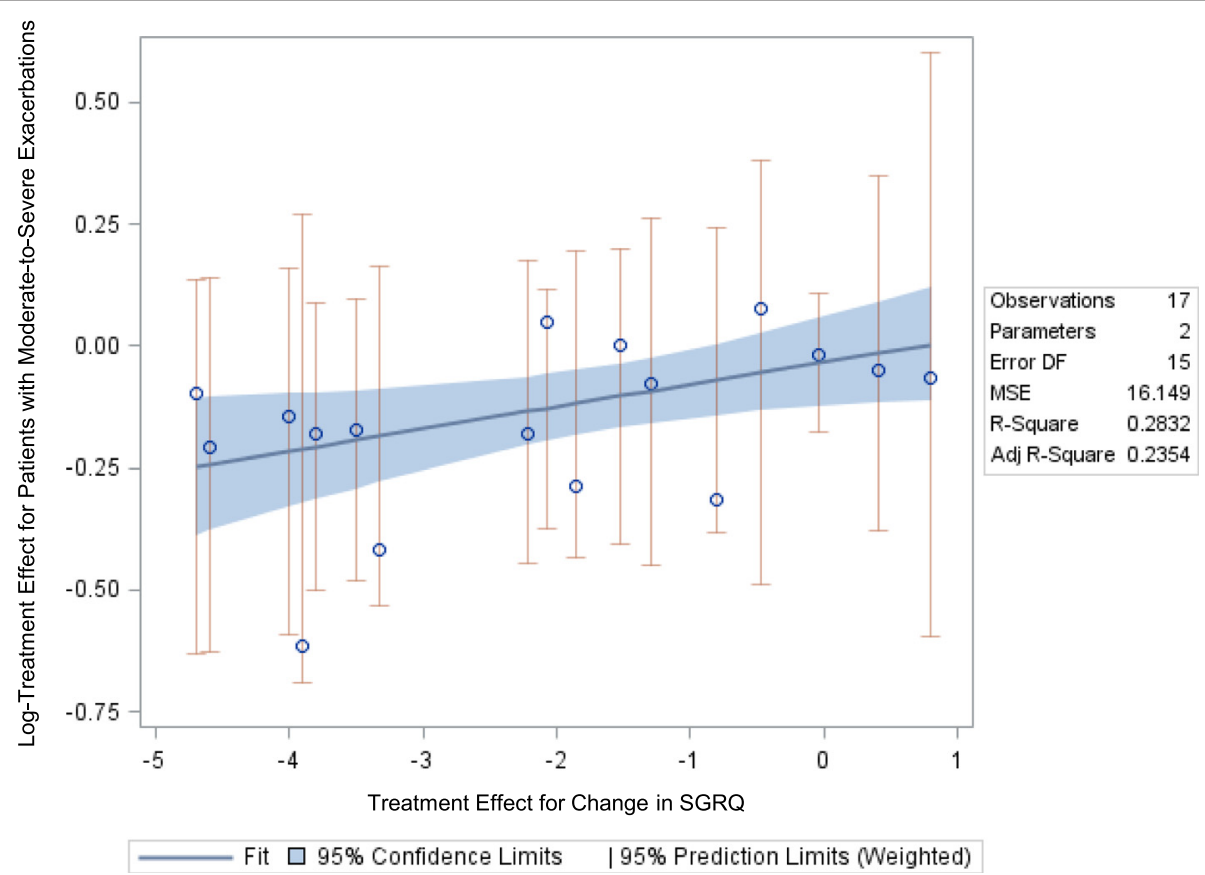

Fig. 5 Relationship between Mean Change in SGRQ Total Score and Risk for a Moderate-to-severe Exacerbation 
Table 4 Study Data for Trials Reporting Mean change in SGRQ Total Score and Patients Experiencing Moderate-to-severe COPD Exacerbation

\begin{tabular}{|c|c|c|c|c|c|c|c|c|}
\hline $\begin{array}{l}\text { Author, } \\
\text { Year }\end{array}$ & Treatment & $\begin{array}{l}\text { Time } \\
\text { point } \\
\text { (weeks) }\end{array}$ & $\begin{array}{l}\mathrm{N} \\
\text { Randomized }\end{array}$ & $\begin{array}{l}\text { Annual } \\
\text { exacerbation } \\
\text { rate (M-S) }\end{array}$ & $\begin{array}{l}\mathrm{N} \text { with M-S } \\
\text { exacerbation }\end{array}$ & $\begin{array}{l}\text { Comparison } \\
\text { data for } \\
\text { Time to first } \\
\text { exacerbation } \\
\text { (Hazard ratio) }\end{array}$ & $\begin{array}{l}\text { Mean change } \\
\text { in SGRQ } \\
\text { Total Score }\end{array}$ & $\begin{array}{l}\text { Comparison } \\
\text { data for SGRQ } \\
\text { (treatment } \\
\text { difference) }\end{array}$ \\
\hline \multirow[t]{2}{*}{$\begin{array}{l}\text { Anzueto, } \\
2009 \text { [23] }\end{array}$} & $\begin{array}{l}\text { Fluticasone propionate/ } \\
\text { salmeterol } 250 \mathrm{mcg} / 50 \mu \mathrm{g} \text { bid }\end{array}$ & 52 & 394 & 1.1 & 208 & $\begin{array}{l}\text { FP250 + S50 vs. } \\
\text { S50: } 0.73\end{array}$ & 2.49 & $\begin{array}{l}\text { FP250+ S50 vs. } \\
\text { S50: }-0.81\end{array}$ \\
\hline & Salmeterol $50 \mu \mathrm{g}$ bid & 52 & 403 & 1.59 & 234 & - & 3.28 & - \\
\hline \multirow[t]{3}{*}{$\begin{array}{l}\text { Bateman, } \\
2010[20]\end{array}$} & $\begin{array}{l}\text { Tiotropium } 5 \mu \mathrm{g} \text { orally inhaled } \\
\text { once daily }\end{array}$ & 48 & 670 & 0.93 & 249 & - & -5.1 & $\begin{array}{l}\text { Tio5 vs. } \\
\text { Placebo: }-3.5\end{array}$ \\
\hline & $\begin{array}{l}\text { Tiotropium } 10 \mu \mathrm{g} \text { orally inhaled } \\
\text { once daily }\end{array}$ & 48 & 667 & 1.02 & 246 & - & -5.5 & $\begin{array}{l}\text { Tio10 vs. } \\
\text { Placebo: }-3.8\end{array}$ \\
\hline & Placebo & 48 & 653 & 1.91 & 288 & - & -1.6 & - \\
\hline \multirow[t]{4}{*}{$\begin{array}{l}\text { Dahl, } 2010 \\
\text { [14] }\end{array}$} & Indacaterol 300 нg & 52 & 437 & 0.6 & 133 & - & -6.5 & $\begin{array}{l}\text { Inda300 vs. } \\
\text { Placebo: }-4.7\end{array}$ \\
\hline & Indacaterol $600 \mu \mathrm{g}$ & 52 & 428 & 0.57 & 116 & - & -7.2 & $\begin{array}{l}\text { Inda600 vs. } \\
\text { Placebo: }-4.6\end{array}$ \\
\hline & Formoterol & 52 & 435 & 0.56 & 126 & - & -7 & F vs. Placebo: -4 \\
\hline & Placebo & 52 & 432 & 0.74 & 145 & - & -1.7 & - \\
\hline \multirow[t]{2}{*}{$\begin{array}{l}\text { Ferguson, } \\
2008[17]\end{array}$} & $\begin{array}{l}\text { Fluticasone propionate/ } \\
\text { salmeterol (FSC) } 250 / 50\end{array}$ & 52 & 394 & 1.06 & 211 & $F P+S$ vs. S: 0.75 & -3.49 & FP/S vs. S: -1.86 \\
\hline & Salmeterol $50 \mu \mathrm{g}$ & 52 & 388 & 1.53 & 230 & - & -1.86 & - \\
\hline \multirow[t]{2}{*}{$\begin{array}{l}\text { Hagedorn, } \\
2013 \text { [33] }\end{array}$} & $\begin{array}{l}\text { Salmeterol xinafoate/ } \\
\text { fluticasone propionate via a } \\
\text { single inhaler (SFC) }\end{array}$ & 52 & 108 & 0.81 & 42 & - & -1.8 & - \\
\hline & $\begin{array}{l}\text { Salmeterol xinafoate/ } \\
\text { fluticasone propionate via } \\
\text { separate inhalers (Sal/FP) }\end{array}$ & 52 & 106 & 0.98 & 44 & - & -2.6 & - \\
\hline \multirow[t]{6}{*}{$\begin{array}{l}\text { Kerwin, } \\
2012[25]\end{array}$} & NVA237 $50 \mu \mathrm{g}$ qd & 52 & 529 & 0.54 & - & $\begin{array}{l}\text { NVA vs. } \\
\text { Placebo: } 0.66\end{array}$ & - & $\begin{array}{l}\text { NVA vs. } \\
\text { Placebo: }-3.32\end{array}$ \\
\hline & Tiotropium $18 \mu \mathrm{g}$ qd & 52 & 268 & & - & NVA vs. Tio: 1.1 & - & $\begin{array}{l}\text { NVA vs. } \\
\text { Tio: }-0.48\end{array}$ \\
\hline & Placebo & 52 & 269 & 0.8 & - & - & - & - \\
\hline & Sharafkhaneh, 2012 [26] & & $\begin{array}{l}\text { Budesonide/ } \\
\text { formoterol } \\
\mathrm{pMDI} 160 / \\
4.5 \mu \mathrm{g} \times 2 \\
\text { inhalations } \\
\text { bid }(320 / 9 \mu \mathrm{g})\end{array}$ & 52 & 407 & 0.867 & 169 & - \\
\hline & & -7.2 & - & & & & & \\
\hline & & & $\begin{array}{l}\text { Budesonide/ } \\
\text { formoterol } \\
\text { pMDI } 80 / \\
4.5 \mu \mathrm{g} \times 2 \\
\text { inhalations } \\
\text { bid }(160 / 9 \mu \mathrm{g})\end{array}$ & 52 & 408 & 0.952 & 173 & - \\
\hline \multirow[t]{2}{*}{-5.5} & - & & & & & & & \\
\hline & $\begin{array}{l}\text { Formoterol DPI } 4.5 \mu \mathrm{g} \\
\times 2 \text { inhalations bid }(9 \mu \mathrm{g})\end{array}$ & 52 & 404 & 1.171 & 182 & - & -5.9 & - \\
\hline \multirow[t]{2}{*}{$\begin{array}{l}\text { Tang, } \\
2013 \text { [27] }\end{array}$} & $\begin{array}{l}\text { Tiotropium } 5 \mu \mathrm{g} \\
(2 \times 2.5 \mu \mathrm{g} / \text { puff })\end{array}$ & 48 & 167 & - & 58 & $\begin{array}{l}\text { Tio5 vs. } \\
\text { Placebo: } 0.54\end{array}$ & -7.1 & $\begin{array}{l}\text { Tio5 vs. } \\
\text { Placebo: }-3.9\end{array}$ \\
\hline & Placebo (2 puffs) & 48 & 171 & - & 83 & - & -3.3 & - \\
\hline
\end{tabular}


Table 4 Study Data for Trials Reporting Mean change in SGRQ Total Score and Patients Experiencing Moderate-to-severe COPD Exacerbation (Continued)

\begin{tabular}{|c|c|c|c|c|c|c|c|c|}
\hline \multirow[t]{2}{*}{$\begin{array}{l}\text { Tashkin, } \\
2008[11]\end{array}$} & $\begin{array}{l}\text { Tiotropium } 18 \mu \mathrm{g} \text { once daily; } \\
\text { followed by } 40 \mu \mathrm{g} \text { of } \\
\text { ipratropium four times daily } \\
\text { for } 30 \text { days after } 4 \text { years of } \\
\text { treatment. }\end{array}$ & 206 & 2987 & - & 2001 & - & -1.25 & - \\
\hline & $\begin{array}{l}\text { Placebo once daily; followed } \\
\text { by } 40 \mu \mathrm{g} \text { of ipratropium four } \\
\text { times daily for } 30 \text { days after } \\
4 \text { years of treatment. }\end{array}$ & 206 & 3006 & - & 2049 & - & -1.21 & - \\
\hline \multirow[t]{2}{*}{$\begin{array}{l}\text { Wedzicha, } \\
2008[34]\end{array}$} & $\begin{array}{l}\text { Salmeterol } 50 \mu \mathrm{g} \text { + fluticasone } \\
\text { propionate } 500 \mu \mathrm{g} \text { bid }\end{array}$ & 104 & 658 & - & 408 & - & -1.7 & - \\
\hline & $\begin{array}{l}\text { Tiotropium bromide } 18 \mu \mathrm{g} \\
\text { once daily }\end{array}$ & 104 & 665 & - & 392 & - & 0.37 & $\begin{array}{l}\text { S + F vs. } \\
\text { Tio18: }-2.07\end{array}$ \\
\hline \multirow[t]{4}{*}{$\begin{array}{l}\text { Jones, } \\
2011[29]\end{array}$} & Aclidinium $200 \mu \mathrm{g}$ & 52 & 627 & - & 167 & $\begin{array}{l}\text { Aclid200 vs. } \\
\text { Placebo } \\
\text { (trial 1): } 1.00\end{array}$ & - & $\begin{array}{l}\text { Aclid200 vs. } \\
\text { Placebo } \\
\text { (trial 1): }-1.53\end{array}$ \\
\hline & Placebo & 52 & 216 & - & 55 & - & - & - \\
\hline & Aclidinium $200 \mu \mathrm{g}$ & 52 & 600 & - & 199 & - & - & $\begin{array}{l}\text { Aclid200 vs. } \\
\text { Placebo } \\
\text { (trial 2): -2.21 }\end{array}$ \\
\hline & Placebo & 52 & 204 & - & 81 & - & - & - \\
\hline
\end{tabular}

$\mathrm{M}-\mathrm{S}=$ moderate-to-severe

$-=$ Not reported

\section{Impact of including All timepoints $>\mathbf{2 4}$ weeks}

Expanding the data set from outcomes reported at $>48$ weeks to include outcomes reported at $>24$ weeks showed similar directionality but weaker results compared with the longterm analysis data of both SGRQ score and $\mathrm{FEV}_{1}$ (data not shown).

\section{Discussion}

Our systematic literature review and regression analysis demonstrated that beneficial mean change in either $\mathrm{FEV}_{1}$ or SGRQ total score was associated with a lower risk for exacerbations. Specifically, it showed that in randomized trials of COPD drug treatments lasting $\geq 48$ weeks, there

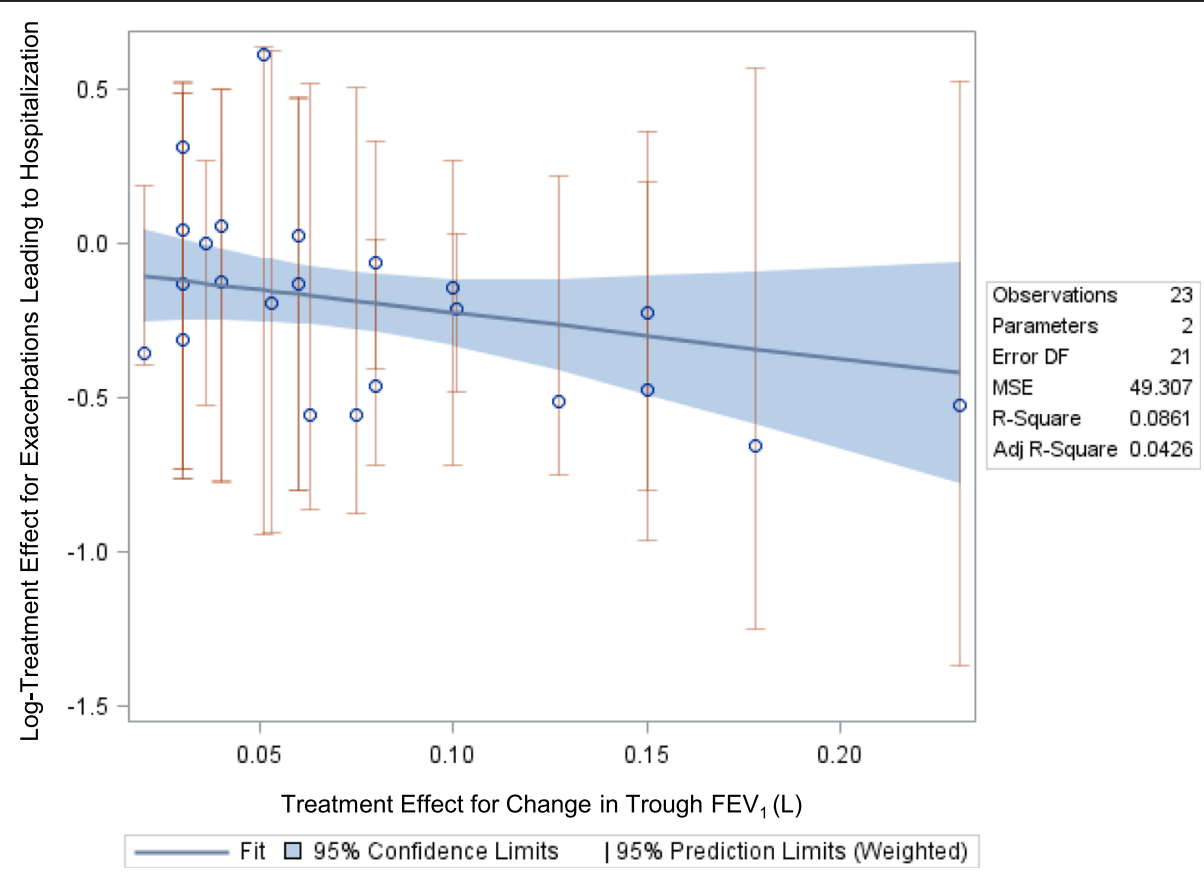

Fig. 6 Relationship between Mean Change in $\mathrm{FEV}_{1}$ and Risk for Hospitalization 


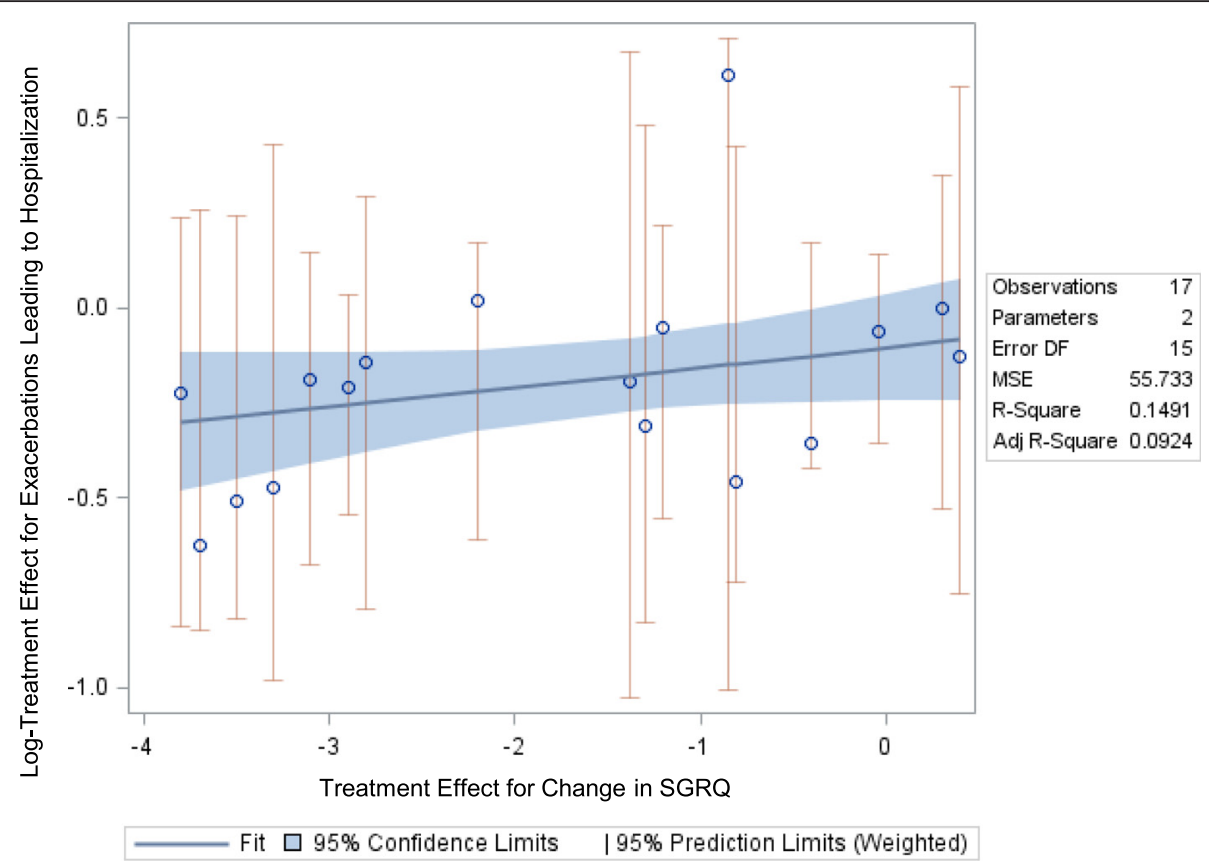

Fig. 7 Relationship between Mean Change in SGRQ and Risk for Hospitalization

was generally a relationship between relative efficacy in improving $\mathrm{FEV}_{1}$ and SGRQ total score and relative efficacy for lowering exacerbation risk. The majority of analyses showed the same trend towards a relationship between positive changes in $\mathrm{FEV}_{1}$ and SGRQ score and exacerbation risk, even though results did not always reach statistical significance. Of note, there was no relationship shown between mean change in $\mathrm{FEV}_{1}$ and annualized exacerbation rate, despite this relationship being moderate and statistically significant when the risk of experiencing at least one exacerbation in patients was analyzed. The mean change in SGRQ total score was not significantly related to the rate of exacerbations across all severities but had a moderate, statistically significant relationship with the rate of moderate-to-severe exacerbations. The relationship between $\mathrm{FEV}_{1}$ and SGRQ score and hospitalizations was less clear, and further research is needed in this area.

To our knowledge, the literature review and regression analysis we conducted is the first such study to evaluate the inter-relationship that health status and lung function have with exacerbation risk. It provides a more rigorous examination of a relationship between laboratory values and exacerbations than has been done in the past, as, unlike former studies, it correlates relative treatment effects instead of absolute ones, thus lowering the possibility of ecological bias. However, as this analysis used only aggregated patient data from published trials, we cannot assume that any statistical association observed between arm-level variables may be translated to patient-level associations. Therefore, our findings cannot be used to predict any outcome at the patient-level. Additionally, our analysis may be limited by the available data for the surrogate measures given the trials reported $\mathrm{FEV}_{1}$ in several different ways. Since our analysis was limited to trough or prebronchodilator $\mathrm{FEV}_{1}$ data, analysis using other measures of $\mathrm{FEV}_{1}$ could yield different results. Similarly, regarding exacerbation severity, we categorized exacerbations based on the definitions reported by study authors using a standardized approach as defined in our methods section. However, in some cases definitions were not reported so we relied on author-defined groupings of any or moderate-to-severe exacerbations.

Our research may have important implications for regulatory assessment of drugs intended to help reduce the risk of exacerbations in COPD and, in particular, the evidence considered in such deliberations. Currently, to gain marketing approval for this indication, such treatments have to be tested in long-term, parallel trials, which represent a logistic and economic burden on the sponsoring organization. Because of this, few trials of COPD drugs are powered to identify a significant difference in the reduced risk of exacerbations. It is for this reason that to date very few drugs have been approved for reducing exacerbations on the basis of prospective 1-2 year parallel trials, usually in patients with history of acute exacerbations in the prior year. Our study suggests changes in $\mathrm{FEV}_{1}$ and SGRQ might serve as reliable surrogate markers of patients' likelihood of experiencing an 
exacerbation. If so, these measures could allow future trials to be shorter and more manageable while still offering key insights into treatments' longer-term efficacy. Since exacerbations can be costly to health plans, payers should consider the effect of medications on these surrogate markers, even when long-term RCTs cannot be carried out. Also, confirmation of our results would broaden the application of data already available from published shorter-term studies. This is especially important since the trials used to inform regulatory approval were powered on each specific drug's expected effect on the acute exacerbation rate and all but one [11] were small and had very selective entry criteria. This contrasts with the trials contributing data for our review and analysis, since these were broader and more inclusive (e.g. with regards to disease duration and reversibility, comorbidities, interventions, and concomitant therapies) and collectively more representative of the general COPD population seen in everyday clinical practice. Therefore, these collated data sources potentially allow more generalizable conclusions to be drawn regarding whether or how standard short-term endpoints assessed in trials relate to effects on exacerbations.

\section{Conclusions}

In conclusion, this study demonstrates a significant association between improvements in $\mathrm{FEV}_{1}$ and SGRQ total score and lower risk for COPD exacerbations. We believe that the results of our study offer providers and payers a more informed picture of the inter-relationship between exacerbations and both $\mathrm{FEV}_{1}$ and SGRQ score, which will aid clinical and formulary decisions while stimulating research questions for future prospective studies.

\section{Abbreviations \\ COPD: chronic obstructive pulmonary disease; $\mathrm{FEV}_{1}$ : forced expiratory volume in one second; HR: hazard ratio; HRU: health resource use; OCS: oral corticosteroids; PICOS: Patient, Interventions, Comparisons, Outcomes, Study Design; RCT: randomized controlled trial; SGRQ: St. George's Respiratory Questionnaire; SLR: systematic literature review.}

\section{Competing interests}

Amber L. Martin, Kyle Fahrbach, Teresa K. Wilcox, and Sarah M. Cadarette are employees of Evidera which received funding from Novartis Pharmaceuticals Corporation to conduct the study on which this manuscript is based. Jessica Marvel is an employee and stockholder of Novartis Pharmaceuticals Corporation.

James F. Donohue is a Member or Chair of the following Data and Safety Monitoring Boards: Teva, Pearl, AZ, Otsuka, Novartis, Insmed, National Institutes of Health and a paid consultant for the following companies; Novartis, GSK, BI, AstraZeneca, Sunovion, Biomark.

\section{Author contributions}

ALM and SMC consulted on the study design, carried out the review, maintained the dataset, and drafted the manuscript. JM formed the research questions and contributed to study design. KF performed the statistical analysis and contributed to data refinement and study design. TKW contributed to study design and helped refine manuscript focus. JFD participated in study design and provided clinical insight. All authors contributed to interpreting the data and read and approved the final manuscript.

\section{Acknowledgements}

The authors would like to acknowledge Abhishek Kavati for his contributions to critical review of the manuscript.

\section{Funding}

Funding for this manuscript was provided by Novartis.

\section{Author details}

${ }^{1}$ Evidera, 430 Bedford Street, Suite 300, 02420 Lexington, MA, USA. ${ }^{2}$ Novartis Pharmaceuticals Corporation, East Hanover, NJ, USA. ${ }^{3}$ University of North

Carolina School of Medicine, Chapel Hill, NC, USA.

Received: 27 November 2015 Accepted: 6 April 2016

Published online: 16 April 2016

\section{References}

1. Halbert RJ, Natoli JL, Gano A, Badamgarav E, Buist AS, Mannino DM. Global burden of COPD: systematic review and meta-analysis. Eur Respir J. 2006;28:523-32

2. Afonso AS, Verhamme KM, Sturkenboom MC, Brusselle GG. COPD in the general population: prevalence, incidence and survival. Respir Med. 2011:105:1872-84

3. Tilert T, Dillon C, Paulose-Ram R, Hnizdo E, Doney B. Estimating the U.S. prevalence of chronic obstructive pulmonary disease using pre- and postbronchodilator spirometry: the National Health and Nutrition Examination Survey (NHANES) 2007-2010. Respir Res. 2013;14:103.

4. Halpin DM, Tashkin DP. Defining disease modification in chronic obstructive pulmonary disease. COPD. 2009;6:211-25.

5. How Is COPD Treated? [http://www.nhlbi.nih.gov/health/health-topics/topics/ copd/treatment]. Accessed 10 Nov 2015.

6. Toy EL, Gallagher KF, Stanley EL, Swensen AR, Duh MS. The economic impact of exacerbations of chronic obstructive pulmonary disease and exacerbation definition: a review. COPD. 2010;7:214-28.

7. Jones PW, Donohue JF, Nedelman J, Pascoe S, Pinault G, Lassen C. Correlating changes in lung function with patient outcomes in chronic obstructive pulmonary disease: a pooled analysis. Respir Res. 2011;12:161.

8. Westwood M, Bourbeau J, Jones PW, Cerulli A, Capkun-Niggli G, Worthy G. Relationship between FEV1 change and patient-reported outcomes in randomised trials of inhaled bronchodilators for stable COPD: A systematic review. Respir Res. 2011:12:40.

9. Johnson KR, Ringland C, Stokes BJ, Anthony DM, Freemantle N, Irs A, Hill SR, Ward RL. Response rate or time to progression as predictors of survival in trials of metastatic colorectal cancer or non-small-cell lung cancer: a metaanalysis. Lancet Oncol. 2006;7:741-6.

10. Bateman ED, Tashkin D, Siafakas N, Dahl R, Towse L, Massey D, Pavia D, Zhong NS. A one-year trial of tiotropium Respimat plus usual therapy in COPD patients. Respir Med. 2010;104:1460-72.

11. Tashkin DP, Celli B, Senn S, Burkhart D, Kesten S, Menjoge S, Decramer M. A 4-year trial of tiotropium in chronic obstructive pulmonary disease. N Engl J Med. 2008;359:1543-54.

12. Calverley PM, Kuna P, Monso E, Costantini M, Petruzzelli S, Sergio F, Varoli G, Papi A, Brusasco V. Beclomethasone/formoterol in the management of COPD: a randomised controlled trial. Respir Med. 2010;104:1858-68.

13. Chapman KR, Rennard SI, Dogra A, Owen R, Lassen C, Kramer B. Long-term safety and efficacy of indacaterol, a long-acting (beta)2-agonist, in subjects with COPD: A randomized, placebo-controlled study. Chest. 2011;140:68-75.

14. Dahl R, Chung KF, Buhl R, Magnussen H, Nonikov V, Jack D, Bleasdale P, Owen R, Higgins M, Kramer B. Efficacy of a new once-daily long-acting inhaled (beta)2-agonist indacaterol versus twice-daily formoterol in COPD. Thorax. 2010;65:473-9.

15. Decramer ML, Chapman KR, Dahl R, Frith P, Devouassoux G, Fritscher C, Cameron R, Shoaib M, Lawrence D, Young D, McBryan D. Once-daily indacaterol versus tiotropium for patients with severe chronic obstructive pulmonary disease (INVIGORATE): A randomised, blinded, parallel-group study. Lancet Respir Med. 2013;1:524-33.

16. Dusser D, Bravo ML, lacono P. The effect of tiotropium on exacerbations and airflow in patients with COPD. Eur Respir J. 2006;27:547-55. 
17. Ferguson GT, Anzueto A, Fei R, Emmett A, Knobil K, Kalberg C. Effect of fluticasone propionate/salmeterol (250/50 microg) or salmeterol (50 microg) on COPD exacerbations. Respir Med. 2008;102:1099-108.

18. van Grunsven $P$, Schermer $T$, Akkermans $R$, Albers $M$, van den Boom $G$, van Schayck O, van Herwaarden C, van Weel C. Short- and long-term efficacy of fluticasone propionate in subjects with early signs and symptoms of chronic obstructive pulmonary disease. Results of the DIMCA study. Respir Med. 2003;97:1303-12.

19. Vincken W, van Noord JA, Greefhorst AP, Bantje TA, Kesten S, Korducki L Cornelissen PJ, Dutch/Belgian Tiotropium Study G. Improved health outcomes in patients with COPD during 1 yr's treatment with tiotropium. Eur Respir J. 2002;19:209-16.

20. Wouters EF, Postma DS, Fokkens B, Hop WC, Prins J, Kuipers AF, Pasma HR, Hensing CA, Creutzberg EC, Group CS. Withdrawal of fluticasone propionate from combined salmeterol/fluticasone treatment in patients with COPD causes immediate and sustained disease deterioration: a randomised controlled trial. Thorax. 2005;60:480-7.

21. Zhou Y, Wang X, Zeng X, Qiu R, Xie J, Liu S, Zheng J, Zhong N, Ran P. Positive benefits of theophylline in a randomized, double-blind, parallel-group, placebo-controlled study of low-dose, slow-release theophylline in the treatment of COPD for 1 year. Respirology. 2006;11:603-10.

22. Dransfield MT, Bourbeau J, Jones PW, Hanania NA, Mahler DA, Vestbo J, Wachtel A, Martinez FJ, Barnhart F, Sanford L, et al. Once-daily inhaled fluticasone furoate and vilanterol versus vilanterol only for prevention of exacerbations of COPD: two replicate double-blind, parallel-group, randomised controlled trials. Lancet Respir Med. 2013;1:210-23.

23. Anzueto A, Ferguson GT, Feldman G, Chinsky K, Seibert A, Emmett A, Knobil K, O'Dell D, Kalberg C, Crater G. Effect of fluticasone propionate/ salmeterol (250/50) on COPD exacerbations and impact on patient outcomes. COPD. 2009;6:320-9.

24. Donohue JF, Niewoehner D, Brooks J, O'Dell D, Church A. Safety and tolerability of once-daily umeclidinium/vilanterol 125/25 mcg and umeclidinium $125 \mathrm{mcg}$ in patients with chronic obstructive pulmonary disease: Results from a 52-week, randomized, double-blind, placebocontrolled study. Respir Res. 2014;15:78.

25. Kerwin E, Hebert J, Gallagher N, Martin C, Overend T, Alagappan VKT, Lu Y, Banerji D. Efficacy and safety of NVA237 versus placebo and tiotropium in patients with COPD: The GLOW2 study. Eur Respir J. 2012;40:1106-14.

26. Sharafkhaneh A, Southard JG, Goldman M, Uryniak T, Martin UJ. Effect of budesonide/formoterol pMDI on COPD exacerbations: a double-blind, randomized study. Respir Med. 2012;106:257-68.

27. Tang Y, Massey D, Zhong NS. Evaluation of the efficacy and safety of tiotropium bromide (5 microg) inhaled via Respimat in Chinese patients with chronic obstructive pulmonary disease. Chin Med J (Engl). 2013;126:3603-7.

28. Calverley PM, Rabe KF, Goehring UM, Kristiansen S, Fabbri LM, Martinez FJ. Roflumilast in symptomatic chronic obstructive pulmonary disease: two randomised clinical trials. Lancet. 2009:374:685-94.

29. Jones PW, Rennard SI, Agusti A, Chanez P, Magnussen H, Fabbri L, Donohue JF, Bateman ED, Gross NJ, Lamarca R, et al. Efficacy and safety of once-daily aclidinium in chronic obstructive pulmonary disease. Respir Res. 2011;12:55.

30. Calverley PM, Boonsawat W, Cseke Z, Zhong N, Peterson S, Olsson H. Maintenance therapy with budesonide and formoterol in chronic obstructive pulmonary disease. Eur Respir J. 2003;22:912-9.

31. Casaburi R, Mahler DA, Jones PW, Wanner A, San PG, ZuWallack RL, Menjoge SS, Serby CW, Witek T, Jr. A long-term evaluation of once-daily inhaled tiotropium in chronic obstructive pulmonary disease. Eur Respir J. 2002;19:217-24.

32. Wedzicha JA, Singh D, Vestbo J, Paggiaro PL, Jones PW, Bonnet-Gonod F, Cohuet G, Corradi M, Vezzoli S, Petruzzelli S, Agusti A. Extrafine beclomethasone/formoterol in severe COPD patients with history of exacerbations. Respir Med. 2014;108:1153-62.

33. Hagedorn C, Kassner F, Banik N, Ntampakas P, Fielder K. Influence of salmeterol/ fluticasone via single versus separate inhalers on exacerbations in severe/very severe COPD. Respir Med. 2013;107:542-9.

34. Wedzicha JA, Calverley PM, Seemungal TA, Hagan G, Ansari Z, Stockley RA, Investigators I. The prevention of chronic obstructive pulmonary disease exacerbations by salmeterol/fluticasone propionate or tiotropium bromide. Am J Respir Crit Care Med. 2008;177:19-26.

\section{Submit your next manuscript to BioMed Central and we will help you at every step:}

- We accept pre-submission inquiries

- Our selector tool helps you to find the most relevant journal

- We provide round the clock customer support

- Convenient online submission

- Thorough peer review

- Inclusion in PubMed and all major indexing services

- Maximum visibility for your research

Submit your manuscript at www.biomedcentral.com/submit
) Biomed Central 